\title{
Phytoextraction with Pteris vittata leads to increased arsenic leaching in soil
}

\author{
CÉLINE PALLUD ${ }^{1}$, SARICK MATZEN ${ }^{2}$, SIRINE FAKRA ${ }^{3}$ \\ AND PETER S NICO ${ }^{3}$
}

${ }^{1}$ UC Berkeley, USA

${ }^{2}$ University of Minnesota

${ }^{3}$ Lawrence Berkeley National Laboratory

Presenting Author: cpallud@berkeley.edu

Anthropogenic enrichment of toxic metal(loid)s, including arsenic (As), in soils poses hazards to humans and ecosystems globally. The arsenic-hyperaccumulating fern Pteris vittata accumulates high concentrations of arsenic in its fronds, and is consequently used to decontaminate soil with minimal site disturbance. However, current remediation times are prohibitively slow. Additionally, heterogeneity in rhizosphere redox conditions affects availability of arsenic. To increase remediation rates, rhizosphere processes mobilizing arsenic for uptake into the fern must be better understood, and possibly optimized through soil treatments that increase arsenic phytoavailability. However, potential arsenic leaching during soil treatment must be constrained.

We combined field-, meso-, and micro-scale studies in three soils (23.5 to $138.0 \mathrm{mg} / \mathrm{kg} \mathrm{As}$ ) to determine the effects of soil fertilization (phosphorus, nitrogen, or compost) or soil inoculation with mycorrhizal fungi on arsenic uptake and leaching in the $P$. vittata soil-plant system. Under field conditions ferns accumulated a mean of $3.6 \%$ of initial soil arsenic, but soil fertilization or inoculation did not affect arsenic uptake rates, which were similar to other published field rates. Additionally, field-scale fern arsenic accumulation across all treatments could not account for $61.5 \%$ of the soil arsenic depletion at depths of $0-20 \mathrm{~cm}$, suggesting that the missing arsenic could have been lost to leaching. Arsenic valence state differed in rhizosphere and bulk soil under field- and meso-scale conditions, suggesting the rhizosphere was a unique reactive zone for arsenic mobilization. More arsenic(III) was found in rhizosphere $(12.8-71.5 \%)$ compared to bulk soils $(<10 \%)$ under field conditions. Under meso-scale conditions, arsenic valence state varied at the micron scale $(2.5-106 \%$ arsenic(III)) in rhizosphere soil and at root surfaces. A meso-scale plant-water mass balance showed uptake of arsenic through transpiration flux accounted for less than $30 \%$ of arsenic accumulated in the fern, suggesting most arsenic taken up into $P$. vittata was mobilized directly in the rhizosphere as a byproduct of nutrient-scavenging processes. Furthermore, arsenic concentrations in leachate increased in the presence of $P$. vittata under meso-scale conditions. We conclude that arsenic phytoextraction is not suitable to remediate these soils, and could even increase environmental arsenic contamination depending on the fate of leached arsenic. 